\title{
Explicit polyhedral approximation of the Euclidean ball
}

\author{
J. Frédéric BONNANS* Marc LEBELLE ${ }^{\dagger}$
}

June 23, 2008

\begin{abstract}
We discuss the problem of computing points of $\mathbb{R}^{n}$ whose convex hull contains the Euclidean ball, and is contained in a small multiple of it. Given a polytope containing the Euclidean ball, we introduce its successor obtained by intersection with all tangent spaces to the Euclidean ball, whose normals point towards the vertices of the polytope. Starting from the $L^{\infty}$ ball, we discuss the computation of the two first successors, and give a complete analysis in the case when $n=6$.
\end{abstract}

\section{Introduction}

Our study is motivated by the need to check if a given ellipsoid $B$, representing a set of possible configurations, is contained in another given set $S$ of "safe" configurations. While the set $S$ is not explicitely known, an oracle can tell if a given point belongs or not to it. A certificate for the inclusion $B \subset S$ is therefore given by a (finite) collection of points $E$ in $S$ whose convex hull denoted $\operatorname{conv}(E)$ contains $B$. After a change of variable (based on the eigenvectors of the quadratic form associated with the ellipsoid $B$ ), we reduce the problem to the case when $B$ is the Euclidean ball.

Calling the oracle has a cost, and so we wish the cardinal of $E$ to be as small as possible. On the other hand, for having good chances that $\operatorname{conv}(E)$ contains $B$, is should be contained in a small multiple of $B$. These two requirements are conflicting, and hence, a reasonable compromise has to be done. We can formalize the discussion as follows:

1. Given $\varepsilon>0$, find if possible a polytope $E$ with "minimal" number of vertices such that

$$
\bar{B} \subset E \subset(1+\varepsilon) \bar{B} .
$$

*INRIA-Saclay and Centre de Mathématiques Appliquées, Ecole Polytechnique, 91128 Palaiseau; Frederic.Bonnans@inria.fr.

†Commissariat à l'Energie Atomique, Direction de la Protection et de la Sûreté Nucléaire, Service Sûreté Nucléaire, Centre de Fontenay aux Roses, B.P. N 6, 92265 Fontenay aux Roses Cedex; marc.lebelle@cea.fr. 
2. (Adaptative method). Compute a sequence $E_{k}$ of polytopes such that $E_{1} \supset E_{2} \supset \cdots \supset B$ and such that $E_{k} \subset\left(1+\varepsilon_{k}\right) \bar{B}$, where $\varepsilon_{k} \downarrow 0$.

The first problem is unfortunately out of reach, so instead we propose an adaptative method based on the following idea. Given a polytope containing the Euclidean ball, consider its refinement obtained by intersecting it with all tangent spaces to the Euclidean ball, whose normals point towards the extreme points of the polytope. We discuss, starting from the $L^{\infty}$ ball, how to compute the two first refinements, and give a complete analysis in the case when $n=6$. A key point in the analysis is the invariance of the refined polytopes under some group of linear transformations in $\mathbb{R}^{n}$ leaving invariant the $L^{\infty}$ ball. So with a given extreme point of a refined polytope is associated the equivalence class obtained as the orbit of this point under the transformations of the group. Specifically, when $n=6$, the first refinement has 480 vertices corresponding to a single orbit, and the second refinement has 5760 vertices corresponding to four orbits.

The paper is organized as follows. Section 2 is devoted to the presentation of some general results and to related problems. Section 3 recalls some properties of polytopes that are invariant under the action of a group of linear transformations. Then in section 4 we make the computation of the first and second reduction of the unit ball in $L^{\infty}$. Finally in the conclusion section we discuss extensions of our resulT.

\section{General results and related problems}

\subsection{Volumes of the $L^{\infty}$ and Euclidean balls}

We recall here some well-known fact about the volumes of the $L^{\infty}$ and Euclidean balls. The easiest polyhedral approximation of the Euclidean ball is the $L^{\infty}$ ball

$$
\bar{B}_{\infty}=\left\{x \in \mathbb{R}^{n} ;\left|x_{i}\right| \leq 1, i=1, \ldots, n\right\} .
$$

This polytope has $2^{n}$ vertices with coordinates \pm 1 . and is included in $\sqrt{n} \bar{B}$. Its volume is $2^{n}$, to be compared with the volume of the Euclidean ball (e.g. Hiriart-Urruty and Pradel [6]) :

$$
\omega_{n}:=2 \frac{\pi^{n / 2}}{n \Gamma(n / 2)},
$$

where $\Gamma(z):=\int_{0}^{\infty} t^{z-1} e^{-t} \mathrm{dd} t$. For any integer $p>1$, it is known that $\Gamma(p)=$ $(p-1)$ !, and hence, $\omega_{2 p}=\pi^{p} / p$ ! In particular, for $n=6$, and so $p=3$, we have

$$
\omega_{6}=\frac{\pi^{3}}{3 !}=\frac{31.006277}{6}=5.1677128
$$

i.e. about one twelwth of the volume of $\bar{B}_{\infty}$. This clearly shows that $\bar{B}_{\infty}$ is not an accurate approximation of the Euclidean ball. 


\subsection{Related problems and approaches}

Ben-Tal and Nemirovski [3] (see also Glineur [5]) considered the problem of outer approximation of the second order cone

$$
C=\left\{(t, x) \in \mathbb{R} \times \mathbb{R}^{n} ; t \geq\|x\|\right\},
$$

by finitely many inequalities. They succeed in obtaining an approximation using a small number of inequalities by using the technique of lifting into a space of larger dimension. For $t=1$ this provides an outer approximation of the Euclidean ball $B$. The number of inequalities is

$$
q+r \leq C\left(n+\log \frac{1}{\varepsilon}\right) .
$$

where $q$ is the number of additional variables, and $r$ is the number of linear constraints.

In principle, finding an outer approximation of the Euclidean ball having a small number of vertices is equivalent to the one of finding an outer approximation having a small number of inequalities. The reason is that with a polytope $P$ whose interior contains zero we can associate its polar set:

$$
P^{*}:=\left\{y \in \mathbb{R}^{n} ; y \cdot x \leq 1, \quad \text { for all } x \in P\right\} .
$$

Clearly, the polar set is another polytope defined by the inequalities

$$
P^{*}:=\left\{y \in \mathbb{R}^{n} ; y \cdot x \leq 1, \quad \text { for all } x \in \operatorname{ext}(P)\right\} .
$$

In addition, $P$ happens to be the polar of $P^{*}$. Therefore the facet defining inequalities of $P$ correspond to the extreme points of $P^{*}$. Now if $P$ is an outer approximation of $B$, contained in $(1+\varepsilon) B$, since polarity is a decreasing mapping (w.r.t. the order of inclusion of sets) and the polar set of $(1+\varepsilon) B$ is $(1+\varepsilon)^{-1} B$, we have that $(1+\varepsilon)^{-1} B \subset P^{*} \subset B$, so that $(1+\varepsilon) P^{*}$ is another outer approximation of $B$, contained in $(1+\varepsilon) B$. Finally we see that if $P$ has a small number of inequalities that after scaling may be written $a^{i} \cdot x \leq 1, i \in I$, then the collection of vectors $\left\{(1+\varepsilon) a^{i} ; i \in I\right\}$ has a convex hull conatined in $(1+\varepsilon) B$ and containing $B$.

We mention also the large literature on the computation of convex hulls, see e.g. the software Quickhull [2]. This could be used for the computation of the intersection of a polyhedron with half spaces.

\section{Invariance of vertices}

Let us first recall some well-known facts about polytopes (see e.g. Nemhauser and al. [8], in particular its chapter 5, and also Nemhauser and Wolsey [7], Schrijver [1]). A polytope $P$ is the convex hull of its finitely many vertices, i.e. points $x$ in $P$ such that $x=\alpha y+(1-\alpha) z$, with $y$ and $z$ in $P$ and $\alpha \in] 0,1[$ 
implies $x=y=z$. The set of vertices of $P$ is denoted $\operatorname{ext}(P)$. We use many times the following property:

$$
\left\{\begin{array}{l}
\text { vertices of a polytope are characterized by the } \\
\text { maximality of the active constraints set. }
\end{array}\right.
$$

By active constraints set at point $x \in P$ we mean, if $P$ is described by a finite number of linear inequalities: $P=\left\{x \in \mathbb{R} ; a_{i} \cdot x \leq b_{i}, 1 \leq i \leq p\right\}$, the set $I(x):=\left\{1 \leq i \leq p ; a_{i} \cdot x=b_{i}\right\}$. Maximality here means that $I(x)$ is maximal with respect to the relation of inclusion of sets (i.e., no other point of the polytope has a set of active constraints different, and including the one of $x$ ). We also know that $P$ is the convex hull of its (finitely many) vertices: $P=\operatorname{conv}(\operatorname{ext}(P))$.

Invariance by a group of transformations. Some polytopes have the property that their vertices are invariant under a set of transformations (i.e., bijections) in $\mathbb{R}^{n}$, and therefore by the group generated by finite compositions of elements of this set and there inverses. For instance, $\bar{B}_{\infty}$ is invariant under permutation of coordinates and sign change of these coordinates.

So let $P$ be a polytope whose set of vertices is invariant under a group $\mathcal{G}$ of transformations. We call (primal) orbit of $x \in \mathbb{R}^{n}$ the image of this point by the group, or under the action of the group :

$$
\mathcal{O}_{x}:=\{G x ; \quad G \in \mathcal{G}\} .
$$

Next assume that the group $\mathcal{G}$ is set of linear mappings. Then the image of $P$ is the convex hull of the one of its vertices. Since the set of the latter is invariant, it follows that $P$ itself is invariant under the action of the group. Consider a valid inequality $(a, b) \in \mathbb{R}^{n} \times \mathbb{R}$ for $P$, i.e., an inequality satisfied by any element of $P$ :

$$
a \cdot x=a_{1} \cdot x_{1}+\cdots a_{n} \cdot x_{n} \leq b, \quad \text { for all } x \in P,
$$

Since $P$ is invariant under the action of the group $\mathcal{G}$, we also have

$$
\left(G^{\top} a\right) \cdot x=a \cdot G x \leq b, \quad \text { for all } x \in P .
$$

Let us call

$$
\mathcal{O}_{a, b}:=\left\{\left(G^{\top} a, b\right) ; \quad G \in \mathcal{G}\right\}
$$

the dual orbit associated with the valid inequality $(a, b)$. To belong to the same (primal ou dual) orbit is an equivalence class.

If $P$ has dimension $n$, we know that a minimal description of $P$ by inequalities is to write one inequality for each facet (faces of maximal dimension $n-1$ ). These facets are the convex hull of their vertices and the set of factes is invariant under the action of the group $\mathcal{G}$. A compact way of describing vertices (facets) is to take one for each equivalence class, and then to compute the corresponding orbits.

Later we will use the following property: if $(a, b)$ is a valid inequality, and $\operatorname{int}(P)$ (the interior of $P$ ) contains 0 , then $b>0$. 
Invariance by permutation or sign change of coordinates In the sequel we consider the particular case of invariance by permutation or sign change of coordinates. The orbit of $x \in \mathbb{R}^{n}$ is therefore the set of points obtained by permutation or sign change of coordinates. It happens that these transformations are orthonormal, and also invariant by transposition. So the dual orbit of $a \in \mathbb{R}^{n}$ is (in the same way) obtained by permutation or sign change of coordinates of $a$.

Denote by

$$
K:=\left\{x \in \mathbb{R}^{n} ; \quad x_{1} \geq x_{2} \geq \cdots \geq x_{n} \geq 0\right\} .
$$

the set of points of $\mathbb{R}^{n}$ with nonnegative and nonincreasing coordinates. Every (primal of dual) orbit contains a unique point in $K$ that we take as the representative of the orbit.

Let $(a, b)$ characterize a facet of $P$. The corresponding representative facet is

$$
\left|a_{\sigma(1)}\right| \cdot x_{1}+\cdots+\left|a_{\sigma(n)}\right| \cdot x_{n} \leq b,
$$

If $x$ (resp. $a$ ) belongs to $K$, the l.h.s. of the linear inequality $a \cdot x \leq b$ is attained over the dual (resp. primal) orbit of $a$ (resp. $x$ ) is maximal when $a$ (resp. $x$ ) also belongs to $K$.

Since an extrem point of $P$ is uniquely determined by its active constraints, it is useful to discuss the latter.

Lemma 3.1 Let $P$ be invariant by permutation or sign change of coordinates, $\bar{x}$ be in $\operatorname{ext}(P) \cap K$, and such that $a \cdot x \leq b$ is a valid inequality for $P$, active at $\bar{x}$ (i.e., $a \cdot \bar{x}=b$ ), where $a \in K$.

Then the active inequality at $\bar{x}$ that belong to the dual orbit $\mathcal{O}_{a, b}$ are of the form

$$
\pm a_{\sigma(1)} x_{1} \pm \cdots \pm a_{\sigma(n)} x_{n} \leq b
$$

where the permutation $\sigma$ is such that there exists a permutation $\pi$ of $\{1, \ldots, n\}$ leaving $\bar{x}$ invariant, i.e., $\bar{x}_{\pi(i)}=\bar{x}_{i}$ for all $i$, and such that $\pi \circ \sigma$ leaves a invariant, and in addition the only changes of sign occur when the product $a_{\sigma(i)} \bar{x}_{i}$ is equal to zero.

Proof. a) Any facet in the orbit of $(a, b)$ can be obtained by permutation of coordinates of $a$, followed by changes of sign for some components. Since both $x$ and $a$ belong to $K$, none of these two transformations can increase the l.h.s. of the linear inequality, and hence, the resulting facet will be active iff each of the two transformations leaves invariant this l.h.s.

b) Let us first consider the action of permutations of coordinates of $a$ :

$$
a_{\sigma(1)} x_{1}+\cdots+a_{\sigma(n)} x_{n} \leq b .
$$

We know [4, Lemma 5.11] that $a \cdot x$ is invariant iff there exists a permutation $\pi$ satisfying the conditions of the lemma (note that the sufficient condition is easily proved). 
c) Obviously changes of sign leave invariant the linear form $a \cdot x$ iff (after having made permutation $\sigma$ ) they operate on coordinates $i$ for which $a_{\sigma(i)}=0$ or $x_{i}=0$.

Remark 3.2 Let us be more explicit about the conditions on permutations $\pi$ and $\sigma$ of the above lemma. We can gather elements of $\{1, \ldots, n\}$ by equivalence classes defined by a common value of de $x_{i}$. Then $\pi$ is any permutation leaving invariant these equivalence classes. Since $\pi \circ \sigma$ leaves $a$ invariant, this means that values of $a$, counted with their multiplicity, must remain invariant in each equivalence class.

Example 3.3 Let $\bar{x}=(1, \alpha, \beta, \beta, \beta, \beta)$, with $1>\alpha>\beta>0$. Then $\pi(1)=1$ and $\pi(2)=2$. If $a \in K$ and $a(1)>a(2)>a(3)$, then $\sigma$ must leave indexes 1 and 2 invariant. Sign changes are possible for the term $a_{\sigma(i)} x_{i}$ when $a_{\sigma(i)}=0$.

\section{Computation of the cuts}

\subsection{First reduction}

With $\bar{x} \in \mathbb{R}^{n} \backslash \bar{B}$, we associate the canonical (sharp) cut separating its projection $\bar{x} /\|\bar{x}\|$ and $\bar{B}$. Since the tangent hyperplane to $\bar{B}$ at $\bar{x} /\|\bar{x}\|$ is orthogonal to $\bar{x}$, the expression of this cut is

$$
c_{\bar{x}}(x):=\bar{x} \cdot x-\|\bar{x}\| \leq 0
$$

Let us denote by $\bar{B}_{\infty}^{1}$ the ball obtained by applying all canonical cuts to all vertices of $\bar{B}_{\infty}$ :

$$
\bar{B}_{\infty}^{1}=\left\{x \in \bar{B}_{\infty} ; c_{y}(x) \leq 0, \text { for all } y=( \pm 1, \ldots, \pm 1)\right\}
$$

Writing the constraints explicitely, we see that $\bar{B}_{\infty}^{1}$ is the intersection of $\bar{B}_{\infty}$ with the closed ball in the $\ell_{1}$ norm, with radius $\sqrt{n}$ :

$$
\bar{B}_{\infty}^{1}=\left\{x \in \bar{B}_{\infty} ; \sum_{i=1}^{n}\left|x_{i}\right| \leq \sqrt{n}\right\} .
$$

We denote by $\mathcal{R}$ the reduction operator that with a polytope containing $\bar{B}$ associates its intersection with all canonical cuts at vertices. In particular

$$
\bar{B}_{\infty}^{1}=\mathcal{R}\left(\bar{B}_{\infty}\right)
$$

Denote by $\lfloor\alpha\rfloor$ the greater integer minorant of $\alpha \in \mathbb{R}$. and by $\operatorname{fr}(\cdot)$ its fractional part: $\operatorname{fr}(\alpha):=\alpha-\lfloor\alpha\rfloor$. We also denote $e_{i}$ the $i$ th basis vector, and 1 the vector of $\mathbb{I R}^{n}$ with coordinates 1 . 
Lemma 4.1 (i) Let $m:=\lfloor\sqrt{n}\rfloor$. A vector $x$ of $\mathbb{R}^{n}$ is a vertex of $\bar{B}_{\infty}^{1}$ iff it has exactly $m$ coordinates equal to \pm 1 , at most $m+1$ nonzero coordinates, and satisfies $\sum_{i=1}^{n}\left|x_{i}\right|=\sqrt{n}$.

(ii) The ball $\bar{B}_{\infty}^{1}$ is contained in the euclidean ball of radius

$$
r^{1}:=\sqrt{\lfloor\sqrt{n}\rfloor+\operatorname{fr}(\sqrt{n})^{2}} \leq n^{1 / 4}
$$

(iii) For $n=6$, the values of constants are

$$
\sqrt{n}=2.4494897 ; \quad m=2 ; \quad \operatorname{fr}(\sqrt{n})=0.4494897 ; \quad r^{1}=1.4839276 .
$$

Proof. (i) Since $\bar{B}_{\infty}^{1}$ is invariant by permutation and sign change of coordinates, vertices of $\bar{B}_{\infty}^{1}$ are obtained by applying these operations to vertices of $\bar{B}_{\infty}^{1} \cap K$.

Let $\bar{x}$ be a vertex of $\bar{B}_{\infty}^{1}$. If $\|\bar{x}\|_{1}<\sqrt{n}$, by (9), the set of its active constraints is maximal in the set of constraints defining $\bar{B}_{\infty}$, and so $\bar{x}$ is a vertex of $\bar{B}_{\infty}$. Since the latter have been eliminated by the cuts, this cannot occur. Therefore $\|\bar{x}\|_{1}=\sqrt{n}$.

If $\bar{x}$ has two coordinates $i$ and $j$ in ]0,1[, set $d=e_{i}-e_{j}$ (difference of basis vectors). Then for $\varepsilon>0$ small enough, the point $x^{\varepsilon}:=\bar{x} \pm \varepsilon d$ belongs to $\bar{B}_{\infty}^{1}$ (since $\left\|x^{\varepsilon}\right\|_{\infty} \leq 1$ and $\left\|x^{\varepsilon}\right\|_{1}=\|\bar{x}\|_{1}=\sqrt{n}$ ) so that $\bar{x}$ is not extremal. We have proved that an extremal point has at most one coordinate outside $\{-1,0,1\}$.

Consequently there exists only one (of course there must exist at least one) vertex $\bar{x}$ in $K$, and then $\bar{x}_{i}=1$, for $i=1$ to $m, x_{m+1}=\operatorname{fr}(\sqrt{n})$, and $\bar{x}_{i}=0$, for $i>m+1$. Since other vertices are obtained by permutation and sign change of coordinates, this proves (i).

(ii) The maximum of the convex function $\|x\|^{2}$ over the polytope $\bar{B}_{\infty}^{1}$ is attained at (at least) one vertex. We conclude with point (i).

(iii) This is a simple application of points (i) and (ii).

It is known that cuts may lead to large increases in the number of vertices, and so it is useful to count the latter.

Lemma 4.2 The polytope $\bar{B}_{\infty}^{1}$ has $2^{m} C_{m}^{n}$ vertices if $n$ is a square, and $2^{m+1}(n-$ $m) C_{m}^{n}$ otherwise. In particular, if $n=6, \bar{B}_{\infty}^{1}$ has 480 points.

Proof. We first count vertices with nonnegative coordinates. There are $C_{m}^{n}$ possible combinations of the $m$ coordinates equal to one among $n$, and if $n$ is not a square, it remains, for each combinations, $n-m$ possibles choices for the fractional coordinate, i.e. $C_{m}^{n}$ vertices if $n$ is a square, and $(n-m) C_{m}^{n}$ otherwise.

The set of all vertices is then obtained by applying arbitrary changes of sign to nonzero coordinates, which gives an additional factor of $2^{m}$ if $n$ is a square, and $2^{m+1}$ otherwise.

\subsection{Second reduction}

We remind that $m:=\lfloor\sqrt{n}\rfloor$. Since vertices of $\bar{B}_{\infty}^{1}$ are known, we may compute the set $\bar{B}_{\infty}^{2}=\mathcal{R}\left(\bar{B}_{\infty}^{1}\right)$ obtained by reduction of $\mathcal{R}\left(\bar{B}_{\infty}^{1}\right)$ : 


$$
\bar{B}_{\infty}^{2}=\left\{x \in \bar{B}_{\infty}^{1} ; c_{y}(x) \leq 0, \text { for all } y \in \operatorname{ext}\left(\bar{B}_{\infty}^{1}\right)\right\} .
$$

Since $\bar{B}_{\infty}^{1}$ is invariant by a sign change of coordinates, this boils down to

$$
\bar{B}_{\infty}^{2}=\left\{x \in \bar{B}_{\infty}^{1} ; \sum_{i=1}^{n} y_{i}\left|x_{i}\right| \leq r^{1}, \text { for all } y \in \operatorname{ext}\left(\bar{B}_{\infty}^{1}\right) \cap \mathbb{R}_{+}^{n}\right\} .
$$

Let $\sigma$ be a permutation of $\{1, \ldots, n\}$ such that $\left|x_{\sigma(i)}\right|$ is nonincreasing. The worst case, in the above inequality, over all permutations of indices is obtained when

$$
y_{\sigma(i)}=1 \text { for } i=1 \text { to } m, y_{\sigma(m+1)}=\operatorname{fr}(\sqrt{n}) \text {, and } y_{\sigma(i)}=0 \text { for } i>m+1 .
$$

In other words, denoting $\varphi:=\operatorname{fr}(\sqrt{n})$, we have that

$$
\bar{B}_{\infty}^{2}=\left\{x \in \bar{B}_{\infty}^{1} ; \sum_{i=1}^{m}\left|x_{\sigma(i)}\right|+\varphi\left|x_{\sigma(m+1)}\right| \leq r^{1}\right\} .
$$

In particular, if $x \in \operatorname{ext}\left(\bar{B}_{\infty}^{2}\right) \cap K$, since necessarily one of the new cuts of type (24) is saturated in $x$, this is the case for the inequality

$$
\sum_{i=1}^{m} x_{i}+\varphi x_{m+1} \leq r^{1}
$$

Lemma 4.3 (i) Any vertex of $\operatorname{ext}\left(\bar{B}_{\infty}^{2}\right) \cap K$ saturates inequality (27).

(ii) In the sequel we assume that $n$ is not a square. Let $x$ belong toext $\left(\bar{B}_{\infty}^{2}\right) \cap K$. Denote $\beta:=x_{m+1}$. Let $m_{0}, m_{1}$ the smallest and biggest indices such that $x_{i}=\beta$ for $m_{0} \leq i \leq m_{1}$. Then the canonical cuts for extremal points of $\bar{B}_{\infty}^{1}$, active at point $x$, are the ones of the form

$$
\begin{gathered}
\sum_{i=1}^{m} x_{i}+\varphi x_{j} \leq r^{1}, \quad m<j \leq m_{1}, \quad \text { if } x_{m}>\beta>0, \\
\sum_{i=1}^{m} x_{i} \pm \varphi x_{j} \leq r^{1}, \quad m<j, \quad \text { if } x_{m}>\beta=0, \\
\sum_{i=1}^{m_{0}-1} x_{i}+\sum_{i \in I} x_{i}+\varphi x_{j} \leq r^{1}, \quad \text { if } x_{m}=\beta>0,
\end{gathered}
$$

with $I \subset\left\{\ell ; m_{0} \leq \ell \leq m_{1}\right\},|I|=m-m_{0}+1, m_{0} \leq j \leq m_{1}, j \notin I$,

$$
\sum_{i=1}^{m_{0}-1} x_{i}+\left(\sum_{i \in I} \pm x_{i}\right) \pm \varphi x_{j} \leq r^{1}, \quad \text { if } x_{m}=\beta=0
$$

with $I \subset\left\{\ell ; m_{0} \leq \ell \leq n\right\},|I|=m-m_{0}+1, m_{0} \leq j \leq n, j \notin I$.

(iii) The point $x$ defined in (ii) has at most one component in $] 0, \beta[\cup] \beta, 1[$.

(iv) If $\beta>0$, and $m_{1}<n$, then $\sum_{i}\left|x_{i}\right|=\sqrt{n}$. 
Proof. (i) Otherwise by (9) it would be a vertex of $\bar{B}_{\infty}^{1}$, whereas the latter are excluded by the cuts.

(ii) Apply remark 3.2 to relation (27). The permutation $\pi$ of c this lemma must (in particular) leave invariant the sets

$$
I_{1}:=\left\{1, \ldots, m_{0}-1\right\} ; \quad I_{2}:=\left\{m_{0}, \ldots, m_{1}\right\} .
$$

In addition, coefficients of indexes 1 to $m_{0}-1$ must be equal to 1 . We distinguish four cases depending on the strictness of the inequalities below:

$$
x_{m} \geq x_{m+1}=\beta \geq 0
$$

a) If both are strict, and hence $m=m_{0}-1$, the permutation $\sigma$ leaves invariants $I_{1}$ and $I_{2}$, without possible sign change of coefficients of the linear form over these two sets, whence (28).

b) If $x_{m}>\beta=0$, the permutation $\sigma$ leaves invariant $I_{1}$, and hence, also $I_{2}=I_{1} \backslash\{1, \ldots, n\}$, and sign changes are possible only over $I_{2}$, whence (29).

c) If $x_{m}=\beta>0$, no sign change can occur, and since coefficients of indices 1 to $m_{0}-1$ are equal to 1 , a coefficient $\varphi$ having to be applied on an index $j \in\left\{m_{0}, \ldots, m_{1}\right\}$, we obtain (30).

d) If $x_{m}=\beta=0$, sign changes are possible for indice not less than $m_{0}$, whence (31).

(iii) If $x$ has two coordinates in ] $\beta, 1\left[\right.$ with indices $i$ and $j$, setting $d=e_{i}-e_{j}$, we have by (ii), $x \pm \varepsilon d \in \bar{B}_{\infty}^{2}$ for $\varepsilon>0$ small enough, giving the desired contradiction. We can exclude in the same way the case when $x$ has two coordinates in $] 0, \beta[$. Finally, let us assume that $x$ has a component $\alpha \in] \beta, 1[$, and another component $\gamma \in] 0, \beta\left[\right.$, of indexes resp. $m_{0}-1$ and $m_{1}+1$. Let $(\delta \alpha, \delta \beta, \delta \gamma)$ be nonzero constants such that

$$
\left\{\begin{array}{l}
\text { (a) } \delta \alpha+\left(m-m_{0}+1+\varphi\right) \delta \beta=0 \\
\text { (b) } \delta \alpha+\left(m_{1}-m_{0}+1\right) \delta \beta+\delta \gamma=0 .
\end{array}\right.
$$

It is easily checked that such a triple exists. Let $d$ be the vector of $\mathbb{R}^{n}$ with zero coordinates, except those with indexes $m_{0}-1, m_{0}$ to $m_{1}$, and $m_{1}+1$, with value resp. $(\delta \alpha, \delta \beta, \delta \gamma)$. We claim that, for $\varepsilon>0$ small, $x^{\varepsilon}:=x \pm \varepsilon d \in \bar{B}_{\infty}^{2}$. Indeed, we have that $\left\|x^{\varepsilon}\right\|_{\infty} \leq 1$, and by (34)(b), $\left\|x^{\varepsilon}\right\|_{1}=\|\bar{x}\|_{1} \leq \sqrt{n}$. Finally the new cuts are satisfied in view of (34)(a) and point (ii) (we are in case (28) or (30)). This gives the desired contradiction.

(iv) If on the countrary $\beta>0, m_{1}<n$ and $\sum_{i}\left|x_{i}\right|<1$, let $j$ such that $\left|x_{j}\right|<\beta$. Then for $\varepsilon>0$ small, $x \pm \varepsilon e_{j} \in \bar{B}_{\infty}^{2}$, and hence, $x$ is not a vertex.

\subsection{Specific results for $n=6$}

Since the explicit computation of vertices in the general case is not easy, let us limit ourself to the case $n=6$. We have established in (23) that $m=2$, $\sqrt{n}=2.4494897, \varphi=\operatorname{fr}(\sqrt{n})=0.4494897, r^{1}=1.4839276$. The vector

$$
\bar{x}^{0}:=(1,1,0.4494897,0,0,0)
$$


is a vertex of $\bar{B}_{\infty}^{1}$, and $\operatorname{ext}\left(\bar{B}_{\infty}^{1}\right)$ is the image of ce point under combinations of permutation and sign changes. We will have to use (9).

In the sequel let us compute all vertices of $\bar{B}_{\infty}^{2}$ in $K$. We again denote $\beta:=x_{m+1}$, and $\alpha, \gamma$ are coordinates (if they exist) of a point $x \in \operatorname{ext}\left(\bar{B}_{\infty}^{2}\right) \cap K$ in $] \beta, 1[$ and $] 0, \beta[$, respectively.

Lemma $4.4 A$ vertex of $\bar{B}_{\infty}^{2}$ in $K$ is of one of the following forms: (superscripts of $\hat{x}$ represent the number of occurences of $(1, \alpha, \beta, \gamma)$ respectively, and $n_{\beta}$ is number of occurences of $\beta$ ):

(i) In the form

$$
\left\{\begin{array}{l}
\hat{x}^{1,1,1,0}=(1, \alpha, \beta, 0,0,0) \\
\hat{x}^{1,1,2,0}=(1, \alpha, \beta, \beta, 0,0) \\
\hat{x}^{1,1,3,0}=(1, \alpha, \beta, \beta, \beta, 0) \\
\hat{x}^{1,1,4,0}=(1, \alpha, \beta, \beta, \beta, \beta)
\end{array}\right.
$$

with $(\alpha, \beta)$ satisfying

$$
1+\alpha+\varphi \beta=r^{1} ; \quad 1+\alpha+n_{\beta} \beta=\sqrt{6} \quad\left(\leq \sqrt{6} \text { if } n_{\beta}=4\right) .
$$

(ii) In the form

$$
\left\{\begin{array}{l}
\hat{x}^{1,0,2,1}=(1, \beta, \beta, \gamma, 0,0) \\
\hat{x}^{1,0,3,1}=(1, \beta, \beta, \beta, \gamma, 0) \\
\hat{x}^{1,0,4,1}=(1, \beta, \beta, \beta, \beta, \gamma)
\end{array}\right.
$$

with $(\beta, \gamma)$ satisfying

$$
1+(1+\varphi) \beta=r^{1} ; \quad 1+n_{\beta} \beta+\gamma=\sqrt{6} .
$$

(iii) In the form

$$
\left\{\begin{array}{l}
\hat{x}^{0,1,2,0}=(\alpha, \beta, \beta, 0,0,0) \\
\hat{x}^{0,1,3,0}=(\alpha, \beta, \beta, \beta, 0,0) ; \\
\hat{x}^{0,1,4,0}=(\alpha, \beta, \beta, \beta, \beta, 0) ; \\
\hat{x}^{0,1,5,0}=(\alpha, \beta, \beta, \beta, \beta, \beta) ;
\end{array}\right.
$$

with $(\alpha, \beta)$ satisfying

$$
\alpha+(1+\varphi) \beta=r^{1} ; \quad \alpha+n_{\beta} \beta=\sqrt{6} \quad\left(\leq \sqrt{6} \text { if } n_{\beta}=5\right) .
$$

(iv) In the form

$$
\left\{\begin{array}{l}
\hat{x}^{0,0,3,1}=(\beta, \beta, \beta, \gamma, 0,0) \\
\hat{x}^{0,0,4,1}=(\beta, \beta, \beta, \beta, \gamma, 0) \\
\hat{x}^{0,0,5,1}=(\beta, \beta, \beta, \beta, \beta, \gamma)
\end{array}\right.
$$

with $(\beta, \gamma)$ satisfying

$$
(2+\varphi) \beta=r^{1} ; \quad n_{\beta} \beta+\gamma=\sqrt{6} .
$$


Proof. a) In view of relation (27), and since $r^{1}<2$, at most one component of vertices in $K$ has value 1 .

b) We show that there is no vertex of $\bar{B}_{\infty}^{2}$ having at most values 0,1 and $\beta$. Indeed, such points would be of the following form (we deduce from points (i) and (iv) of lemma 4.3 relations (45) and (48)) :

b1) If $x$ has no component equal to 1 , the possible cases are

$$
\left\{\begin{array}{l}
\hat{x}^{0,0,3,0}=(\beta, \beta, \beta, 0,0,0) \\
\hat{x}^{0,0,4,0}=(\beta, \beta, \beta, \beta, 0,0) \\
\hat{x}^{0,0,5,0}=(\beta, \beta, \beta, \beta, \beta, 0) \\
\hat{x}^{0,0,6,0}=(\beta, \beta, \beta, \beta, \beta, \beta)
\end{array}\right.
$$

and then $\beta$ and $n_{\beta}$ must satisfy

$$
\begin{cases}(2+\varphi) \beta & =r^{1} ; \\ n_{\beta} \beta & =\sqrt{6} \quad\left(\leq \sqrt{6} \text { if } n_{\beta}=6\right) .\end{cases}
$$

We deduce that $\beta=r^{1} /(2+\varphi)=0.6058109$, and so

$$
\begin{cases}n_{\beta}=\frac{\sqrt{6}}{\beta}=4.043324 & \text { if } n_{\beta} \leq 5, \\ n_{\beta} \beta=6 \beta=3.6348654>\sqrt{6} & \text { if } n_{\beta}=6,\end{cases}
$$

in contradiction with either the first, or the second relation of (45).

b2) If only one component is equal to 1 , the possible cases are

$$
\left\{\begin{array}{l}
\hat{x}^{1,0,2,0}=(1, \beta, \beta, 0,0,0) \\
\hat{x}^{1,0,3,0}=(1, \beta, \beta, \beta, 0,0) \\
\hat{x}^{1,0,4,0}=(1, \beta, \beta, \beta, \beta, 0) \\
\hat{x}^{1,0,5,0}=(1, \beta, \beta, \beta, \beta, \beta)
\end{array}\right.
$$

and then $\beta$ and $n_{\beta}$ must satisfy

$$
1+(1+\varphi) \beta=r^{1} ; \quad 1+n_{\beta} \beta=\sqrt{6} .
$$

It follows that $\beta=\left(r^{1}-1\right) /(1+\varphi)=0.3338606$, and so

$$
\begin{cases}n_{\beta}=\frac{\sqrt{6}-1}{\beta}=4.3416012 & \text { if } n_{\beta} \leq 4, \\ n_{\beta} \beta=5 \beta=1.6693032>\sqrt{6}-1=1.4494897 & \text { if } n_{\beta}=5,\end{cases}
$$

in contradiction with either the first, or the seconde relation of (48).

c) It remains to check the other cases of lemma 4.3. If the first component of $x$ equals 1 , then $\alpha$ or $\gamma$ may present, which correspond to cases (i) and (ii). Similarly, if the first component of $x$ is less than one, it is equal either to $\alpha$ or to $\beta$, and in the last case $\gamma$ may be present, which corresponds to cases (iii) and (iv). 
We now show that only four cases do occur among the possible values in lemma 4.4. All other cases will be eliminated thanks to relations

$$
\begin{cases}\text { a) } & 1>\alpha \\ \text { b) } & \alpha>\beta \\ \text { c) } & \beta>\gamma \\ \text { d) } & \gamma>0\end{cases}
$$

Theorem 4.5 The only cases that occur, among those stated in lemma 4.4, satisfying (50), are the points (which indeed have norm greater than one):

$$
\left\{\begin{array}{llll}
\hat{x}^{1,1,4,0} & \text { with } & \alpha=0.3616887, & \beta=0.2719503 \\
\hat{x}^{1,0,4,1} & \text { with } & \beta=0.3338606, & \gamma=0.1140472 \\
\hat{x}^{0,1,4,0} & \text { with } & \alpha=0.9351854, & \beta=0.3785761 \\
\hat{x}^{0,0,4,1} & \text { with } & \beta=0.6058109, & \gamma=0.0262462
\end{array}\right.
$$

with coordinates

$\left\{\begin{array}{llllll}1.0000000 & 0.3616887 & 0.2719503 & 0.2719503 & 0.2719503 & 0.2719503 \\ 1.0000000 & 0.3338606 & 0.3338606 & 0.3338606 & 0.3338606 & 0.1140472 \\ 0.9351854 & 0.3785761 & 0.3785761 & 0.3785761 & 0.3785761 & 0.0000000 \\ 0.6058109 & 0.6058109 & 0.6058109 & 0.6058109 & 0.0262462 & 0.0000000\end{array}\right.$

Proof. We consider cases (i) to (iv) of lemma 4.4.

Case (i) Solving (37) gives

\begin{tabular}{|c|c|c|}
\hline$n_{\beta}$ & $\alpha$ & $\beta$ \\
\hline 1 & -0.3044506 & 1.7539404 \\
2 & 0.2040131 & 0.6227383 \\
3 & 0.3137615 & 0.3785761 \\
\hline
\end{tabular}

Conditions (50) are not satisfied since $\alpha<\beta$. Finally for $n_{\beta}=4$ we solve with equality in (37). The values of $\alpha$ and $\beta$, displayed in the first row of (51), satisfy (50). Using (9), we see that for $n_{\beta}=4$ these are the only possible values of $\alpha$ and $\beta$. In addition the norm of vector $\mathrm{a}$ is 1.1944231 .

Case (ii) Solving (39) gives

\begin{tabular}{|c|c|c|}
\hline$n_{\beta}$ & $\beta$ & $\gamma$ \\
\hline 2 & 0.3338606 & 0.7817685 \\
3 & 0.3338606 & 0.4479078 \\
4 & 0.3338606 & 0.1140472 \\
\hline
\end{tabular}

Conditions (50) are therefore not satisfied for $\beta$ equal to 2 and 3 , while they are for $n_{\beta}=4$, with a vector norm equal to 1.2078321 .

In the case $n_{\beta}=5$, we still have $\beta=0.3338606$, and so

$$
1+n_{\beta} \beta=2.669303>\sqrt{6}=2.4494897
$$

so that (39) has no solution.

Case (iii) Solving (41) gives donne 


\begin{tabular}{|c|c|c|}
\hline$n_{\beta}$ & $\alpha$ & $\beta$ \\
\hline 2 & -1.058391 & 1.7539404 \\
3 & 0.5812747 & 0.6227383 \\
\hline
\end{tabular}

Conditions (50) are therefore not satisfied. For $n_{\beta}=4$, the values of $\alpha$ and $\beta$, displayed in the third row of (51), satisfy (50). In addition the vector norm is 1.2032669 .

In the case $n_{\beta}=5$, by difference of relations in (41) (with inequality for the second one) we obtain

$$
\left(n_{\beta}-1-\varphi\right) \beta \leq \sqrt{6}-r^{1},
$$

i.e., with $n_{\beta}=5,3.5505103 \beta \leq 0.9655622$, and so $\beta \leq \beta^{M}:=0.2719503$. So again with (41)

$$
\alpha \geq r^{1}-(1+\varphi) \beta^{M} \geq 1.0897385,
$$

in contradiction with $\alpha<1$.

Case (iv) Solving (43) gives (here $\beta$ always has the same value)

\begin{tabular}{|c|c|c|}
\hline$n_{\beta}$ & $\beta$ & $\gamma$ \\
\hline 3 & 0.6058109 & 0.6320571 \\
4 & 0.6058109 & 0.0262462 \\
5 & 0.6058109 & -0.5795647 \\
\hline
\end{tabular}

So only the case $n_{\beta}=4$ satisfies (50). In addition the norm of the vector is 1.211906 .

Remark 4.6 Since only the value of $\beta$ is repeated, and is repeated four times, the number of distinct points obtained by permutations of one of the four vectors is $6 ! / 4 !=30$. For each of them the number of points with distinct coordinates is $2^{6}$ in absence of zeros, and $2^{5}$ in the presence of a single zero. The total number is therefore $30 \times\left(2 \times 2^{6}+2 \times 2^{5}\right)=5760$.

We have proved that vertices of $\bar{B}_{\infty}^{2}$ in $K$ are among the four points stated in theorem 4.5. Let us now check that these four points are really vertices of $\bar{B}_{\infty}^{2}$

Lemma 4.7 The four points stated in theorem 4.5 are vertices of $\bar{B}_{\infty}^{2}$.

Proof. a) It one of these four points is not extremal, then it should be a convex combination of the union of the equivalent class of the three other points. Since each of the four points attains the maximal possible value for the sum of components over $\bar{B}_{\infty}^{2}$, which is $\sqrt{6}$, if one of the four points is not extremal, then it is a convex combination of the vertices obtained by permutation only (and not sign changes) of the three others.

b) Each of the two first points having for first component 1, which is the maximal possible value, if not extremal, must be a convex combination of the points 
obtained by a permutation of indexes of the other one, leaving the first component invariant. Now the first (resp. second) one attains the maximum (resp. minimum) of components different of one. They can therefore not be convex combinations of the set of points obtained by permutation of the other one.

c) Similarly, the two last points being the only ones with zero components, each of them, if it is convex combination of the set of points obtained by permutation of the other one, is also a convex combination for permutations that leave the last component invariant. Yet the third (resp. fourth) attains the maximal (resp. minimal) value of nonzero components. They therefore must both be extremal.

\section{Conclusion}

Motivated by some applications, we have made the computation of the second refinement of the Euclidean ball, starting from the $L^{\infty}$ ball, in $\mathbb{R}^{6}$. It seems that the results can be extended to dimensions less than 6 without much difficulty.

An interesting extension of this approach would be the use of formal computation systems in order to give a "formal" guarantee of correctness, and possibly to extend to other dimensions, this result.

Another useful direction would be a totally adaptative approach in which only vertices than happen not to belong to the unknown convex set $S$ are cut. clearly we then loose the invariance properties. This would be of great help in case when the oracle is expensive (it may be the results of a long simulation, or possibly a physical experiment).

\section{References}

[1] Schrijver A. Theory of linear and integer programming. Wiley, 1986.

[2] C.B. Barber, D.P. Dobkin, and H. Huhdanpaa. The quickhull algorithm for convex hulls. ACM Transactions on Mathematical Software, 22(4):469-483, 1996.

[3] A. Ben-Tal and A. Nemirovski. On polyhedral approximations of the secondorder cone. Mathematics of Operations Research, 26(2):193-205, 2001.

[4] J.F. Bonnans. Optimisation Continue. Dunod, Paris, 2006.

[5] F. Glineur. Computational experiments with a linear approximation of second-order cone optimization. Faculté Polytechnique de Mons, 2000.

[6] J.B. Hiriart-Urruty and M. Pradel. Les boules. Quadrature, 2004.

[7] G. Nemhauser and L. Wolsey. Integer and combinatorial optimization. Wiley-Interscience Series in Discrete Mathematics and Optimization. John Wiley \& Sons Inc., New York, 1999. Reprint of the 1988 original, A WileyInterscience Publication. 
[8] G.L. Nemhauser, A.H.G. Rinnoy Kan, and M.J. Todd, editors. Optimization, volume 1 of Handbooks in Operations Research and Management Science. North-Holland, Amsterdam, 1989. 